\title{
Generalized Qualification and Qualification Levels for Spectral Regularization Methods
}

\author{
T. Herdman, R. D. Spies and K. G. Temperini
}

\begin{abstract}
The concept of qualification for spectral regularization methods (SRM) for inverse ill-posed problems is strongly associated to the optimal order of convergence of the regularization error ([2], [5], [6], [11]). In this article, the definition of qualification is extended and three different levels are introduced: weak, strong and optimal. It is shown that the weak qualification extends the definition introduced by Mathé and Pereverzev ([6]), mainly in the sense that the functions associated to orders of convergence and source sets need not be the same. It is shown that certain methods possessing infinite classical qualification, e.g. truncated singular value decomposition (TSVD), Landweber's method and Showalter's method, also have generalized qualification leading to an optimal order of convergence of the regularization error. Sufficient conditions for a SRM to have weak qualification are provided and necessary and sufficient conditions for a given order of convergence to be strong or optimal qualification are found. Examples of all three qualification levels are provided and the relationships between them as well as with the classical concept of qualification and the qualification introduced in [6] are shown. In particular, SRMs having extended qualification in each one of the three levels and having zero or infinite classical qualification are presented. Finally several implications of this theory in the context of orders of convergence, converse results and maximal source sets for inverse ill-posed problems, are shown.
\end{abstract}

Keywords. Qualification, Regularization method, Inverse ill-posed problem.

This work was supported by DARPA/SPO, NASA LaRC and the National Institute of Aerospace under grant VT-03-1, 2535, and in part by AFOSR Grants F49620-03-1-0243 and FA9550-07-1-0273, by Consejo Nacional de Investigaciones Científicas y Técnicas, CONICET, and by Universidad Nacional del Litoral, U.N.L., Argentina, through project CAI+D 2006, P.E. 236.

T. Herdman, Interdisciplinary Center for Applied Mathematics, ICAM, Virginia Tech, Blacksburg, VA 24061, USA. E-mail: herdman@icam.vt.edu

R. D. Spies, Instituto de Matemática Aplicada del Litoral, IMAL, CONICET-UNL, Güemes 3450, S3000GLN, Santa Fe, Argentina. Departamento de Matemática, Facultad de Ingeniería Química, UNL, Santa Fe, Argentina. E-mail: rspies@imalpde.santafe-conicet.gov.ar

K. G. Temperini, IMAL, CONICET-UNL, Güemes 3450, S3000GLN, Santa Fe, Argentina. Departamento de Matemática, Facultad de Humanidades y Ciencias, UNL, Santa Fe, Argentina. E-mail: ktemperini@sanatfe-conicet.gov.ar 


\section{Introduction and preliminaries}

Let $X, Y$ be infinite dimensional Hilbert spaces and $T: X \rightarrow Y$ a bounded linear operator. If $\mathcal{R}(T)$, the range of $T$, is not closed it is well known that the linear operator equation

$$
T x=y
$$

is ill-posed, in the sense that $T^{\dagger}$, the Moore-Penrose generalized inverse of $T$, is not bounded [2]. The Moore-Penrose generalized inverse is strongly related to the leastsquares (LS) solutions of (1). In fact equation (1) has a LS solution if and only if $y$ belongs to $\mathcal{D}\left(T^{\dagger}\right)$, the domain of $T^{\dagger}$, which is defined as $\mathcal{D}\left(T^{\dagger}\right) \doteq \mathcal{R}(T) \oplus \mathcal{R}(T)^{\perp}$. In that case, $x^{\dagger} \doteq T^{\dagger} y$ is the best approximate solution (i.e. the LS solution of minimum norm) and the set of all LS solutions of (1) is given by $x^{\dagger}+\mathcal{N}(T)$. If the problem is ill-posed, then $x^{\dagger}$ does not depend continuously on the data $y$. Hence if instead of the exact data $y$, only an approximation $y^{\delta}$ is available, with $\left\|y-y^{\delta}\right\| \leq \delta$, where $\delta>0$ is the noise level or observation error, then it is possible that $T^{\dagger} y^{\delta}$ does not exist or, if it exists, then it will not necessarily be a good approximation of $x^{\dagger}$, even if $\delta$ is very small. This instability becomes evident when trying to approximate $x^{\dagger}$ by standard numerical methods and procedures. Thus, for instance, except under rather restrictive conditions ([4], [12]), the application of the standard LS approximations procedure on a sequence $\left\{X_{n}\right\}$ of finite dimensional subspaces of $X$, whose union is dense in $X$, will result in a sequence $\left\{x_{n}\right\}$ of LS approximating solutions which does not converge to $x^{\dagger}$ (see [9]). Moreover, this divergence can occur with arbitrarily large speed (see [10]).

Ill-posed problems must be regularized before pretending to successfully attack the problem of numerically approximating their solutions. Regularizing an ill-posed problem such as (1) essentially means approximating the operator $T^{\dagger}$ by a parametric family of continuous operators $\left\{R_{\alpha}\right\}$, where $\alpha$ is called the regularization parameter. More precisely, for $\alpha \in\left(0, \alpha_{0}\right)$ with $\alpha_{0} \in(0,+\infty]$, let $R_{\alpha}: Y \rightarrow X$ be a continuous (not necessarily linear) operator. The set $\left\{R_{\alpha}\right\}_{\alpha \in\left(0, \alpha_{0}\right)}$ is said to be a "family of regularization operators" (FRO) for $T^{\dagger}$, if for every $y \in \mathcal{D}\left(T^{\dagger}\right)$, there exists a parameter choice rule $\alpha=\alpha\left(\delta, y^{\delta}\right)$ such that

$$
\lim _{\delta \rightarrow 0^{+}} \sup _{\substack{y^{\delta} \in Y \\\left\|y^{\delta}-y\right\| \leq \delta}}\left\|R_{\alpha\left(\delta, y^{\delta}\right)} y^{\delta}-T^{\dagger} y\right\|=0 .
$$

Here the parameter choice rule $\alpha: \mathbb{R}^{+} \times Y \rightarrow\left(0, \alpha_{0}\right)$ is such that

$$
\lim _{\delta \rightarrow 0^{+}} \sup _{\substack{y^{\delta} \in Y \\\left\|y^{\delta}-y\right\| \leq \delta}} \alpha\left(\delta, y^{\delta}\right)=0 .
$$

If $y \in \mathcal{D}\left(T^{\dagger}\right)$, then $x^{\dagger}$ satisfies the normal equation $\left(T^{*} T\right) x^{\dagger}=T^{*} y$ and $x^{\dagger}$ can be written as

$$
x^{\dagger} \doteq T^{\dagger} y=\int_{0}^{\|T\|^{2}+} \frac{1}{\lambda} d E_{\lambda} T^{*} y
$$


where $\left\{E_{\lambda}\right\}_{\lambda \in \mathbb{R}}$ is the spectral family associated to the self-adjoint operator $T^{*} T$ (see [1], [2]). However, since we are assuming that $\mathcal{R}(T)$ is not closed (and therefore $\mathcal{D}\left(T^{\dagger}\right) \subsetneq Y$ ), if $y \notin \mathcal{D}\left(T^{\dagger}\right)$ then the integral in (2) does not exist since in that case $0 \in \sigma\left(T^{*} T\right)$ and $\frac{1}{\lambda}$ has a pole at 0 . Moreover in this case, the operator $T^{\dagger}$ defined in (2) for $y \in \mathcal{D}\left(T^{\dagger}\right)$, is not bounded. For that reason, many regularization methods are based on spectral theory and consist on defining $R_{\alpha} \doteq \int_{0}^{\|T\|^{2}+} g_{\alpha}(\lambda) d E_{\lambda} T^{*}$ where $\left\{g_{\alpha}\right\}$ is a family of functions appropriately defined such that for every $\lambda \in\left(0,\|T\|^{2}\right]$ there holds $\lim _{\alpha \rightarrow 0^{+}} g_{\alpha}(\lambda)=\frac{1}{\lambda}$.

Let $\left\{g_{\alpha}\right\}_{\alpha \in\left(0, \alpha_{0}\right)}$ be a parametric family of functions $g_{\alpha}:[0,+\infty) \rightarrow \mathbb{R}$ defined for all $\alpha \in\left(0, \alpha_{0}\right)$. We shall say that $\left\{g_{\alpha}\right\}_{\alpha \in\left(0, \alpha_{0}\right)}$ is a "spectral regularization method" (SRM), if it satisfies the following hypotheses:

H1. For every fixed $\alpha \in\left(0, \alpha_{0}\right), g_{\alpha}(\lambda)$ is piecewise continuous with respect to $\lambda$, for $\lambda \in[0,+\infty)$

H2. There exists a constant $C>0$ (independent of $\alpha$ ) such that $\left|\lambda g_{\alpha}(\lambda)\right| \leq C$ for every $\lambda \in[0,+\infty)$;

H3. For every $\lambda \in(0,+\infty), \lim _{\alpha \rightarrow 0^{+}} g_{\alpha}(\lambda)=\frac{1}{\lambda}$.

It can be shown that if $\left\{g_{\alpha}\right\}_{\alpha \in\left(0, \alpha_{0}\right)}$ is a SRM then the family of operators $\left\{R_{\alpha}\right\}_{\alpha \in\left(0, \alpha_{0}\right)}$ defined by

$$
R_{\alpha} \doteq \int g_{\alpha}(\lambda) d E_{\lambda} T^{*}=g_{\alpha}\left(T^{*} T\right) T^{*}
$$

is a FRO for $T^{\dagger}\left([2]\right.$, Theorem 4.1). In this case we shall say that $\left\{R_{\alpha}\right\}_{\alpha \in\left(0, \alpha_{0}\right)}$ is a "spectral regularization family" for $T^{\dagger}$. The use of this terminology has to do with the fact that each one of its elements is defined in terms of an integral with respect to the spectral family $\left\{E_{\lambda}\right\}_{\lambda \in \mathbb{R}}$ associated to the operator $T^{*} T$. Note that given the operator $T$, it is sufficient that $g_{\alpha}(\lambda)$ be defined for $\lambda \in\left[0,\|T\|^{2}\right]$, since $E_{\lambda}$ is "constant" outside that interval.

It is well known that for ill-posed problems it is not possible to reconstruct the exact solution $x^{\dagger}$ with any degree of accuracy unless additional a-priori information about $x^{\dagger}$ is available ([10], [2] Proposition 3.11). On the other hand, given certain $a$ priori information about $x^{\dagger}$, it could be desirable to know the best order of convergence (of the regularization error $\left\|R_{\alpha} y-x^{\dagger}\right\|$ as a function of the regularization parameter $\alpha$, or of the total error $\left\|R_{\alpha} y^{\delta}-x^{\dagger}\right\|$ as a function of the noise level $\delta$ ), that can be achieved with a regularization method under those a-priori assumptions. Conversely, given an order of convergence, one could be interested in determining the possible existence of "source sets" on which a certain regularization method reaches that order of convergence. In this case it could further be of interest to determine "maximal source sets". All these problems are strongly related to the concepts of qualification and saturation of a regularization method ([2], [3], [5], [6], [7], [8]).

In [11] the notion of qualification of a regularization method was introduced for the first time and the decisive role of this concept in relation to the order of convergence of the regularization error was shown. In the sequel, we shall simply denote with $\left\{g_{\alpha}\right\}$ the SRM $\left\{g_{\alpha}\right\}_{\alpha \in\left(0, \alpha_{0}\right)}$. We now recall the definition of classical qualification for SRMs (see 
[2]).

Definition 1.1. Let $\left\{g_{\alpha}\right\}$ be a SRM and denote with $\mathcal{I}\left(g_{\alpha}\right)$ the set

$\mathcal{I}\left(g_{\alpha}\right) \doteq\left\{\mu \geq 0: \forall \lambda \in[0,+\infty), \exists k>0\right.$ such that $\left.\lambda^{\mu}\left|1-\lambda g_{\alpha}(\lambda)\right| \leq k \alpha^{\mu}, \forall \alpha \in\left(0, \alpha_{0}\right)\right\}$

and let $\mu_{0} \doteq \sup _{\mu \in \mathcal{I}\left(g_{\alpha}\right)} \mu$. If $0<\mu_{0}<+\infty$, we say that $\left\{g_{\alpha}\right\}$ has classical qualification and in that case the number $\mu_{0}$ is called "order" of the classical qualification.

Remark 1.1. Note that $0 \in \mathcal{I}\left(g_{\alpha}\right)$ by virtue of H2 and therefore $\mathcal{I}\left(g_{\alpha}\right)$ is always nonempty.

In [6] Mathé and Pereverzev first introduced the following definition of qualification for a spectral regularization method, formalizing and extending the classical notion of the concept.

Definition 1.2. Let $\rho:(0, a] \rightarrow(0, \infty)$ be an increasing function. It is said that the regularization method $\left\{g_{\alpha}\right\}$ has qualification $\rho$ if there exists a constant $\gamma \in(0, \infty)$ such that

$$
\sup _{\lambda \in(0, a]}\left|1-\lambda g_{\alpha}(\lambda)\right| \rho(\lambda) \leq \gamma \rho(\alpha) \quad \forall \alpha \in(0, a]
$$

In this article we generalize the previous concept, mainly by allowing the function $\rho(\lambda)$ appearing in the left hand side of (3) to be substituted by a general function $s(\lambda)$ with similar properties.

Remark 1.2. It is important to point out that in [2] the "classical qualification" of a method was defined to be the number $\mu_{0}$ in Definition 1.1 (even in the case $\left.\mu_{0}=\infty\right)$. However, from our point of view the "generalized qualification" of a method will not be a number but rather a function of the regularization parameter $\alpha$ as an order of convergence in the sense of Definition 1.2. In the case of SRMs with classical qualification of positive finite order $\mu_{0}$, the corresponding generalized qualification will be shown to be the function $\rho(\alpha)=\alpha^{\mu_{0}}$, coinciding with the classical approach. Since in the extreme cases $\mu_{0}=0$ and $\mu_{0}=\infty$ that function does not define an order of convergence, we have preferred to exclude them from the definition of classical qualification (Definition 1.1) and, accordingly, we shall say that the method does not have classical qualification.

The organization of this article is as follows. In Section 2 the concepts of weak and strong source-order pair and of order-source pair are defined and three qualification levels for SRM are introduced: weak, strong and optimal. A sufficient condition for the existence of weak qualification is provided and necessary and sufficient conditions for an order of convergence to be strong or optimal qualification are given. In Section 3, examples of all qualification levels are provided and the relationships between them and with the classical qualification and the qualification introduced in [6] are shown. In particular, SRMs having qualification in each one of the three levels and not having classical qualification are presented. Finally several implications of this theory in the context of orders of convergence, converse results and maximal source sets for inverse ill-posed problems are shown in Section 4. 


\section{Source-order and order-source pairs. Generalized qualification and qualification levels.}

It is well known that there exist SRMs for which the corresponding $\mu_{0}$ given in Definition 1.1 is infinity, e.g. truncated singular value decomposition (TSVD), Landweber's method and Showalter's method. However, a careful analysis leads to observe that the concept of qualification as optimal order of convergence of the regularization error remains alive underlying most of these and many other methods. In this section we generalize the definition of qualification introduced by Mathé-Pereverzev in [6] and thereby the notion of classical qualification of a SRM. Also three different levels of qualification are introduced: weak, strong and optimal. These levels introduce natural hierarchical categories for the SRMs and we show that the generalized qualification corresponds to the lowest of these levels. Moreover, a sufficient condition which guarantees that a SRM possesses qualification in the sense of this generalization is provided and necessary and sufficient conditions for a given order of convergence to be strong or optimal qualification are found.

We denote with $\mathcal{O}$ the set of all non decreasing functions $\rho: \mathbb{R}^{+} \rightarrow \mathbb{R}^{+}$such that $\lim _{\alpha \rightarrow 0^{+}} \rho(\alpha)=0$ and with $\mathcal{S}$ the set of all continuous functions $s: \mathbb{R}_{0}^{+} \rightarrow \mathbb{R}_{0}^{+}$satisfying $s(0)=0$ and such that $s(\lambda)>0$ for every $\lambda>0$. If moreover $s$ is increasing, then it is an index function in the sense of Mathé-Pereverzev ([6]).

Definition 2.1. Let $\rho, \tilde{\rho} \in \mathcal{O}$. We say that " $\rho$ precedes $\tilde{\rho}$ at the origin" and we denote it with $\rho \preceq \tilde{\rho}$, if there exist positive constants $c$ and $\varepsilon$ such that $\rho(\alpha) \leq c \tilde{\rho}(\alpha)$ for every $\alpha \in(0, \varepsilon)$.

Definition 2.2. Let $\rho, \tilde{\rho} \in \mathcal{O}$. We say that " $\rho$ and $\tilde{\rho}$ are equivalent at the origin" and we denote it with $\rho \approx \tilde{\rho}$, if they precede each other at the origin, that is, if there exist constants $\varepsilon, c_{1}, c_{2}, \varepsilon>0,0<c_{1}<c_{2}<\infty$ such that $c_{1} \rho(\alpha) \leq \tilde{\rho}(\alpha) \leq c_{2} \rho(\alpha)$ for every $\alpha \in(0, \varepsilon)$.

Clearly, " $\approx$ " introduces an order of equivalence in $\mathcal{O}$. Analogous definitions and notation will be used for $s, \tilde{s} \in \mathcal{S}$.

Definition 2.3. Let $\left\{g_{\alpha}\right\}$ be a $S R M, r_{\alpha}(\lambda) \doteq 1-\lambda g_{\alpha}(\lambda), \rho \in \mathcal{O}$ and $s \in \mathcal{S}$.

i) We say that $(s, \rho)$ is a "weak source-order pair for $\left\{g_{\alpha}\right\}$ " if it satisfies

$$
\frac{s(\lambda)\left|r_{\alpha}(\lambda)\right|}{\rho(\alpha)}=O(1) \quad \text { for } \alpha \rightarrow 0^{+}, \forall \lambda>0 .
$$

ii) We say that $(s, \rho)$ is a "strong source-order pair for $\left\{g_{\alpha}\right\}$ " if it is a weak sourceorder pair and there is no $\lambda>0$ for which $O(1)$ in (4) can be replaced by o(1). That is, if (4) holds and also

$$
\limsup _{\alpha \rightarrow 0^{+}} \frac{s(\lambda)\left|r_{\alpha}(\lambda)\right|}{\rho(\alpha)}>0 \quad \forall \lambda>0 .
$$


iii) We say that $(\rho, s)$ is an "order-source pair for $\left\{g_{\alpha}\right\}$ " if there exist a constant $\gamma>0$ and a function $h:\left(0, \alpha_{0}\right) \rightarrow \mathbb{R}^{+}$with $\lim _{\alpha \rightarrow 0^{+}} h(\alpha)=0$, such that

$$
\frac{s(\lambda)\left|r_{\alpha}(\lambda)\right|}{\rho(\alpha)} \geq \gamma \quad \forall \lambda \in[h(\alpha),+\infty) .
$$

In the previous definitions we shall refer to the function $\rho$ as the "order of convergence" and to $s$ as the "source function". The reason for using this terminology will become clear in Section 4 when we shall see applications of these concepts in the context of direct and converse results for regularization methods.

The following observations follow immediately from the definitions.

(i) If $(s, \rho)$ is a weak source-order pair for $\left\{g_{\alpha}\right\}$ which is not a strong source-order pair, then there exists $\lambda_{0}>0$ such that $\lim \sup \frac{s\left(\lambda_{0}\right)\left|r_{\alpha}\left(\lambda_{0}\right)\right|}{\rho(\alpha)}=0$ and therefore $(\rho, s)$ cannot be an order-source pair for $\left\{g_{\alpha}\right\}$. Thus if $(\rho, s)$ is an order-source pair and $(s, \rho)$ is a weak source-order pair, then $(s, \rho)$ is further a strong source-order pair in the sense of $\boldsymbol{i i})$.

(ii) Let $\rho, \tilde{\rho} \in \mathcal{O}$.

(a) If $(s, \rho)$ is a weak source-order pair for $\left\{g_{\alpha}\right\}$ and $\rho \preceq \tilde{\rho}$ then $(s, \tilde{\rho})$ is also a weak source-order pair for $\left\{g_{\alpha}\right\}$.

(b) If $(s, \rho)$ is a weak source-order pair for $\left\{g_{\alpha}\right\}$ and $\tilde{s} \in \mathcal{S}$ is such that there exists $c>0$ for which $\tilde{s}(\lambda) \leq c s(\lambda)$ for every $\lambda>0$, then $(\tilde{s}, \rho)$ is also a weak source-order pair for $\left\{g_{\alpha}\right\}$.

In the following definition we introduce the concept of generalized qualification and three different levels of it.

Definition 2.4. Let $\left\{g_{\alpha}\right\}$ be a SRM.

i) We say that $\rho$ is "weak or generalized qualification of $\left\{g_{\alpha}\right\}$ " if there exists a function s such that $(s, \rho)$ is a weak source-order pair for $\left\{g_{\alpha}\right\}$.

ii) We say that $\rho$ is "strong qualification of $\left\{g_{\alpha}\right\}$ " if there exists a function s such that $(s, \rho)$ is a strong source-order pair for $\left\{g_{\alpha}\right\}$.

iii) We say that $\rho$ is "optimal qualification of $\left\{g_{\alpha}\right\}$ " if there exists a function $s$ such that $(s, \rho)$ is a strong source-order pair for $\left\{g_{\alpha}\right\}$ (it is sufficient that $(s, \rho)$ be a weak source-order pair) and $(\rho, s)$ is an order-source pair for $\left\{g_{\alpha}\right\}$.

It is important to observe that weak qualification generalizes the concept of qualification introduced by Mathé and Pereverzev in [6] and therefore, the notion of classical qualification. In fact, if $\left\{g_{\alpha}\right\}$ has continuous qualification $\rho(\alpha)$ in the sense of Definition 1.2 and $\lim _{\alpha \rightarrow 0^{+}} \rho(\alpha)=0$, then the function

$$
\tilde{\rho}(\alpha) \doteq \begin{cases}0, & \text { si } \alpha=0 \\ \rho(\alpha), & \text { si } 0<\alpha \leq a \\ \rho(a), & \text { si } \alpha>a\end{cases}
$$


is weak qualification of $\left\{g_{\alpha}\right\}$. However, these two notions are not equivalent. We shall see later on that it is possible for a function to be weak qualification of a SRM and not be qualification according to Definition 1.2 (see comments at the end of Section 3).

It is timely to note here that if $\left\{g_{\alpha}\right\}$ has classical qualification of order $\mu_{0}$, then $\rho(\alpha)=\alpha^{\mu}$ is weak qualification of $\left\{g_{\alpha}\right\}$ and moreover $\left(\lambda^{\mu}, \alpha^{\mu}\right)$ is a weak source-order pair for $\left\{g_{\alpha}\right\}$ for every $\mu \in\left(0, \mu_{0}\right]$. Conversely, if for $\mu>0,\left(\lambda^{\mu}, \alpha^{\mu}\right)$ is a weak source-order pair for $\left\{g_{\alpha}\right\}$, then this method has classical qualification (of order $\mu_{0} \geq \mu$ ) provided that $\mu_{0} \doteq \sup \left\{\mu:\left(\lambda^{\mu}, \alpha^{\mu}\right)\right.$ is a weak source-order pair for $\left.\left\{g_{\alpha}\right\}\right\}<+\infty$.

The following result provides a sufficient condition for the existence of weak qualification of a SRM.

Theorem 2.1. Let $\left\{g_{\alpha}\right\}$ be a SRM such that for every fixed $\lambda>0, g_{\alpha}(\lambda)$ is decreasing in $\alpha$, for $\alpha \in\left(0, \alpha_{0}\right)$.

a) If there exist an increasing function $h:\left(0, \alpha_{0}\right) \rightarrow \mathbb{R}^{+}$with $\lim _{\alpha \rightarrow 0^{+}} h(\alpha)=0, \rho^{*} \in \mathcal{O}$ and $\varepsilon>0$ such that for every $\alpha \in(0, \varepsilon)$,

$$
\sup _{\lambda \in[h(\alpha),+\infty)}\left|r_{\alpha}(\lambda)\right| \leq \rho^{*}(\alpha),
$$

then $\left\{g_{\alpha}\right\}$ has weak qualification and in that case $\rho^{*}$ is weak qualification of the method.

b) If for every $\alpha \in\left(0, \alpha_{0}\right), r_{\alpha}(\lambda)$ is positive and monotone decreasing for $\lambda \in$ $(0,+\infty)$, then it is always possible to find $h$ and $\rho^{*}$ as in a) satisfying (8) for all $\alpha \in\left(0, \alpha_{0}\right)$.

Proof. a) Let $h:\left(0, \alpha_{0}\right) \rightarrow \mathbb{R}^{+}$be an increasing function with $\lim _{\alpha \rightarrow 0^{+}} h(\alpha)=0, \rho^{*} \in \mathcal{O}$ and $\varepsilon>0$ such that for every $\alpha \in(0, \varepsilon)$ condition (8) holds.

Case I: there exists $\tilde{\alpha} \in(0, \varepsilon)$ such that $\sup _{\lambda \in[h(\tilde{\alpha}),+\infty)}\left|r_{\tilde{\alpha}}(\lambda)\right|>0$.

Since $h(\alpha)$ is increasing, it follows that $\sup _{\lambda \in[h(\alpha),+\infty)}\left|r_{\alpha}(\lambda)\right|>0$ for every $\alpha \in(0, \tilde{\alpha}]$. Let $\lambda_{0}>0$. Then for every $\alpha \in(0, \tilde{\alpha}]$,

$$
\frac{\left|r_{\alpha}\left(\lambda_{0}\right)\right|}{\rho^{*}(\alpha)} \leq \frac{\left|r_{\alpha}\left(\lambda_{0}\right)\right|}{\sup _{\lambda \in[h(\alpha),+\infty)}\left|r_{\alpha}(\lambda)\right|} .
$$

Since $\lim _{\alpha \rightarrow 0^{+}} h(\alpha)=0$, there exists $\alpha^{*} \in(0, \tilde{\alpha})$ such that $\lambda_{0} \in[h(\alpha),+\infty)$ for every $\alpha \in\left(0, \alpha^{*}\right]$, from which it follows that for every $\alpha \in\left(0, \alpha^{*}\right]$,

$$
\frac{\left|r_{\alpha}\left(\lambda_{0}\right)\right|}{\sup _{\lambda \in[h(\alpha),+\infty)}\left|r_{\alpha}(\lambda)\right|} \leq 1 \text {. }
$$

¿From (9) and (10) it follows that for every $\lambda_{0}>0$

$$
\limsup _{\alpha \rightarrow 0^{+}} \frac{\left|r_{\alpha}\left(\lambda_{0}\right)\right|}{\rho^{*}(\alpha)} \leq 1 .
$$

Then, for any bounded $s \in \mathcal{S}$ the pair $\left(s, \rho^{*}\right)$ satisfies (4), i.e., it is a weak source-order pair for $\left\{g_{\alpha}\right\}$. Thus we have proved that $\rho^{*}$ is weak qualification of $\left\{g_{\alpha}\right\}$. 
Case II: $\sup _{\lambda \in[h(\alpha),+\infty)}\left|r_{\alpha}(\lambda)\right|=0$ for every $\alpha \in(0, \varepsilon)$.

Let $\lambda_{0}>0$. Since $\lim _{\alpha \rightarrow 0^{+}} h(\alpha)=0$, there exists $\alpha^{*} \in(0, \varepsilon)$ such that $\lambda_{0} \in[h(\alpha),+\infty)$ for every $\alpha \in\left(0, \alpha^{*}\right]$. Then $\left|r_{\alpha}\left(\lambda_{0}\right)\right| \leq \sup _{\lambda \in[h(\alpha),+\infty)}\left|r_{\alpha}(\lambda)\right|=0$ for every $\alpha \in\left(0, \alpha^{*}\right)$, from what it follows that $r_{\alpha}\left(\lambda_{0}\right)=0$. Then, for any $s \in \mathcal{S}$,

$$
\frac{s\left(\lambda_{0}\right) r_{\alpha}\left(\lambda_{0}\right)}{\rho^{*}(\alpha)}=0 \text { for all } \alpha \in\left(0, \alpha^{*}\right)
$$

Therefore, $\left(s, \rho^{*}\right)$ is a weak source-order pair for $\left\{g_{\alpha}\right\}$, which implies that $\rho^{*}$ is weak qualification of $\left\{g_{\alpha}\right\}$. (Note that in this case any $\rho^{*} \in \mathcal{O}$ is weak qualification of $\left\{g_{\alpha}\right\}$.)

b) Let $\left\{g_{\alpha}\right\}$ be a SRM such that for every $\alpha \in\left(0, \alpha_{0}\right), r_{\alpha}(\lambda)$ is positive and monotone decreasing for $\lambda \in(0,+\infty)$. For $\lambda>0$ we define $f(\lambda) \doteq\left(1-e^{-\lambda}\right) \theta(\lambda)$, where

$$
\theta(\lambda) \doteq \sup \left\{\gamma \in\left(0, \alpha_{0}\right): r_{\alpha}(\lambda) \leq \lambda \forall \alpha \in(0, \gamma)\right\}
$$

Since for every $\lambda>0, \lim _{\alpha \rightarrow 0^{+}} r_{\alpha}(\lambda)=0$, it follows that given $\lambda>0$ there exists $\gamma=\gamma(\lambda)>0$ such that $r_{\alpha}(\lambda) \leq \lambda$ for every $\alpha \in(0, \gamma)$. Then $\theta(\lambda) \neq-\infty$, moreover $\theta(\lambda) \in\left(0, \alpha_{0}\right]$ for every $\lambda>0$ and therefore, $f(\lambda) \in\left(0, \alpha_{0}\right)$ for every $\lambda>0$. On the other hand, since for every $\alpha \in\left(0, \alpha_{0}\right), r_{\alpha}(\lambda)$ is decreasing for $\lambda>0$, it follows immediately that $f$ is strictly increasing. Furthermore, since $f$ is bounded, it has countably many jump discontinuity points. Therefore, it is possible to assume, without loss of generality, that $f$ is continuous (since, if it is not, we can redefine it in such a way that it be continuous, by subtracting the jumps at the discontinuity points).

Thus $f: \mathbb{R}^{+} \rightarrow\left(0, \alpha_{0}\right)$ is continuous, strictly increasing with $\lim _{\lambda \rightarrow 0^{+}} f(\lambda)=0$. Therefore, its inverse function $f^{-1}$ exists over the range of $f$ and it is strictly increasing and continuous with $\lim _{\alpha \rightarrow 0^{+}} f^{-1}(\alpha)=0$. It is possible to extend $f^{-1}$ to $\left(0, \alpha_{0}\right)$ in such a way that it preserves all these properties. We shall denote with $h$ this extension.

For $\alpha \in\left(0, \alpha_{0}\right)$, we define $z(\alpha) \doteq \sup _{\lambda \in[h(\alpha),+\infty)}\left|r_{\alpha}(\lambda)\right|=r_{\alpha}(h(\alpha))$. Since for every $\alpha \in\left(0, \alpha_{0}\right), r_{\alpha}(\lambda)$ is positive for all $\lambda>0$, it follows that $z(\alpha)$ is also positive. Since for every $\lambda>0, f(\lambda)<\theta(\lambda)$, the definition of $\theta(\lambda)$ implies that $r_{f(\lambda)}(\lambda) \leq \lambda$ for every $\lambda>0$, or equivalently, $r_{\alpha}(h(\alpha)) \leq h(\alpha)$ for every $\alpha \in\left(0, \alpha_{0}\right)$. Then $0<z(\alpha) \leq h(\alpha)$ for every $\alpha \in\left(0, \alpha_{0}\right)$ and the fact that $\lim _{\alpha \rightarrow 0^{+}} h(\alpha)=0$ implies that $\lim _{\alpha \rightarrow 0^{+}} z(\alpha)=0$. If further $z$ is a non decreasing function, then $z \in \mathcal{O}$ and it suffices to define $\rho^{*} \doteq z$. On the contrary, since $z$ is bounded and positive with $\lim _{\alpha \rightarrow 0^{+}} z(\alpha)=0$, there always exists a function $\rho^{*} \in \mathcal{O}$ such that $z(\alpha) \leq \rho^{*}(\alpha)$ for every $\alpha \in\left(0, \alpha_{0}\right)$, as we wanted to show.

¿From the previous Theorem, it follows that the SRMs $\left\{g_{\alpha}\right\}$ such that for every $\lambda>0, g_{\alpha}(\lambda)$ is decreasing for $\alpha \in\left(0, \alpha_{0}\right)$ and for every $\alpha \in\left(0, \alpha_{0}\right), r_{\alpha}(\lambda)$ is positive and decreasing for $\lambda>0$, do possess weak qualification. It is important to observe that most of the usual SRMs do in fact satisfy these conditions. In particular this is so for Landweber's and Showalter's methods. 
Now given the SRM $\left\{g_{\alpha}\right\}$ and $\rho \in \mathcal{O}$, we define

$$
s_{\rho}(\lambda) \doteq \liminf _{\alpha \rightarrow 0^{+}} \frac{\rho(\alpha)}{\left|r_{\alpha}(\lambda)\right|} \quad \text { for } \quad \lambda \geq 0 .
$$

Note that $s_{\rho}(0)=0$.

In the next three results we will see that the characteristics of a given function $\rho \in \mathcal{O}$, as a possible strong or optimal qualification of a SRM, can be determined from properties of that function $s_{\rho}$.

Proposition 2.1. (Necessary and sufficient condition for strong qualification.) A function $\rho \in \mathcal{O}$ such that $s_{\rho} \in \mathcal{S}$ is strong qualification of $\left\{g_{\alpha}\right\}$ if and only if

$$
0<s_{\rho}(\lambda)<+\infty \text { for every } \lambda>0 \text {. }
$$

Proof. Suppose that $\rho$ is strong qualification of $\left\{g_{\alpha}\right\}$. Then there exists a function $s \in \mathcal{S}$ such that $(s, \rho)$ is a strong source-order pair for $\left\{g_{\alpha}\right\}$. Then, for every $\lambda>0$,

$$
s_{\rho}(\lambda)=\liminf _{\alpha \rightarrow 0^{+}} \frac{\rho(\alpha)}{\left|r_{\alpha}(\lambda)\right|}=\frac{1}{\limsup _{\alpha \rightarrow 0^{+}} \frac{\left|r_{\alpha}(\lambda)\right|}{\rho(\alpha)}}=\frac{s(\lambda)}{\limsup _{\alpha \rightarrow 0^{+}} \frac{s(\lambda)\left|r_{\alpha}(\lambda)\right|}{\rho(\alpha)}} .
$$

Thus (12) follows from (4) and (5).

Conversely, suppose now that $0<s_{\rho}(\lambda)<+\infty$ for every $\lambda>0$. We will show that $\rho$ is strong qualification of $\left\{g_{\alpha}\right\}$. For that let us see that $\left(s_{\rho}, \rho\right)$ is a strong source-order pair for $\left\{g_{\alpha}\right\}$. Since $0<s_{\rho}(\lambda)<+\infty$ for every $\lambda>0$, it follows that

$$
\limsup _{\alpha \rightarrow 0^{+}} \frac{s_{\rho}(\lambda)\left|r_{\alpha}(\lambda)\right|}{\rho(\alpha)}=s_{\rho}(\lambda) \limsup _{\alpha \rightarrow 0^{+}} \frac{\left|r_{\alpha}(\lambda)\right|}{\rho(\alpha)}=1 \quad \forall \lambda>0 .
$$

Then, $s_{\rho}$ verifies (4) and (5), which, together with the fact that $s_{\rho} \in \mathcal{S}$, implies that $\left(s_{\rho}, \rho\right)$ is a strong source-order pair and thus $\rho$ is strong qualification of $\left\{g_{\alpha}\right\}$.

Proposition 2.2. Let $\rho \in \mathcal{O}$ be strong qualification of $\left\{g_{\alpha}\right\}$ and $s \in \mathcal{S}$. Then $(s, \rho)$ is a strong source-order pair for $\left\{g_{\alpha}\right\}$ if and only if there exists $k>0$ such that $s(\lambda) \leq k s_{\rho}(\lambda)$ for every $\lambda>0$.

Proof. Since $\rho$ is strong qualification, by Proposition 2.1 it follows that $s_{\rho}(\lambda)>0$ for every $\lambda>0$. Suppose now that $(s, \rho)$ is a strong source-order pair for $\left\{g_{\alpha}\right\}$. Then there exist positive constants $k$ and $\varepsilon$ such that $\frac{s(\lambda)\left|r_{\alpha}(\lambda)\right|}{\rho(\alpha)} \leq k$ for every $\lambda>0, \alpha \in(0, \varepsilon)$. Then, for every $\lambda>0$

$$
\frac{s(\lambda)}{s_{\rho}(\lambda)}=s(\lambda) \limsup _{\alpha \rightarrow 0^{+}} \frac{\left|r_{\alpha}(\lambda)\right|}{\rho(\alpha)}=\limsup _{\alpha \rightarrow 0^{+}} \frac{s(\lambda)\left|r_{\alpha}(\lambda)\right|}{\rho(\alpha)} \leq k,
$$

and therefore $s(\lambda) \leq k s_{\rho}(\lambda)$ for every $\lambda>0$.

Conversely, suppose that there exists $k>0$ such that $s(\lambda) \leq k s_{\rho}(\lambda)$ for every $\lambda>0$. Since $s_{\rho}(\lambda)>0$, it then follows that

$$
k \geq \frac{s(\lambda)}{s_{\rho}(\lambda)}=\limsup _{\alpha \rightarrow 0^{+}} \frac{s(\lambda)\left|r_{\alpha}(\lambda)\right|}{\rho(\alpha)} \quad \forall \lambda>0,
$$


that is, $(s, \rho)$ is a weak source-order pair for $\left\{g_{\alpha}\right\}$. Moreover since $s(\lambda)$ and $s_{\rho}(\lambda)$ are positive for all $\lambda>0$, it follows that $s(\lambda)$ verifies (5) and therefore $(s, \rho)$ is, furthermore, a strong source-order pair for $\left\{g_{\alpha}\right\}$.

Theorem 2.2. (Necessary and sufficient condition for optimal qualification.) A function $\rho \in \mathcal{O}$ such that $s_{\rho} \in \mathcal{S}$ is optimal qualification of $\left\{g_{\alpha}\right\}$ if and only if $s_{\rho}$ verifies (6) and (12).

Proof. Suppose that $\rho$ is optimal qualification. Then $\rho$ is strong qualification and it follows from Proposition 2.1 that $s_{\rho}$ verifies (12). Moreover since $\rho$ is optimal qualification, there exists $s \in \mathcal{S}$ such that $(s, \rho)$ is a strong source-order pair and $(\rho, s)$ is an order-source pair. From the latter it follows that there exist a constant $\gamma>0$ and a function $h:\left(0, \alpha_{0}\right) \rightarrow \mathbb{R}^{+}$with $\lim _{\alpha \rightarrow 0^{+}} h(\alpha)=0$, such that

$$
\frac{s(\lambda)\left|r_{\alpha}(\lambda)\right|}{\rho(\alpha)} \geq \gamma \quad \forall \lambda \in[h(\alpha),+\infty) .
$$

On the other hand, since $(s, \rho)$ is a strong source-order pair for $\left\{g_{\alpha}\right\}$, it follows from Proposition 2.2 that there exists $k>0$ such that

$$
s(\lambda) \leq k s_{\rho}(\lambda) \text { for every } \lambda>0 .
$$

¿From (13) and (14) it follows that

$$
\frac{s_{\rho}(\lambda)\left|r_{\alpha}(\lambda)\right|}{\rho(\alpha)} \geq \frac{\gamma}{k} \quad \forall \lambda \in[h(\alpha),+\infty),
$$

that is, $s_{\rho}$ satisfies (6) as we wanted to show.

Conversely, suppose that $s_{\rho} \in \mathcal{S}$ verifies (6) and (12). By Proposition 2.1 we have that $\left(s_{\rho}, \rho\right)$ is a strong source-order pair for $\left\{g_{\alpha}\right\}$ and (6) implies that $\left(\rho, s_{\rho}\right)$ is an order-source pair. Then, $\rho$ is optimal qualification of $\left\{g_{\alpha}\right\}$.

Next we will show the uniqueness of the source function.

Theorem 2.3. If $\rho$ is optimal qualification of $\left\{g_{\alpha}\right\}$ then there exists at most one function $s$ (in the sense of the equivalence classes induced by Definition 2.2) such that $(s, \rho)$ is a strong source-order pair and $(\rho, s)$ is an order-source pair for $\left\{g_{\alpha}\right\}$. Moreover if $s_{\rho} \in \mathcal{S}$, then $s_{\rho}$ is such a unique function.

Proof. Given that $\rho$ is optimal qualification of $\left\{g_{\alpha}\right\}$, there exists at least one function $s$ such that $(s, \rho)$ is a strong source-order pair and $(\rho, s)$ is an order-source pair for $\left\{g_{\alpha}\right\}$. Suppose now that there exist $s_{1}$ and $s_{2}$ such that $\left(s_{1}, \rho\right)$ and $\left(s_{2}, \rho\right)$ are strong sourceorder pairs and $\left(\rho, s_{1}\right)$ and $\left(\rho, s_{2}\right)$ are order-source pairs for $\left\{g_{\alpha}\right\}$. Then there exist $\gamma>0$ and a function $h:\left(0, \alpha_{0}\right) \rightarrow \mathbb{R}^{+}$with $\lim _{\alpha \rightarrow 0^{+}} h(\alpha)=0$, such that $\frac{s_{2}(\lambda)\left|r_{\alpha}(\lambda)\right|}{\rho(\alpha)} \geq \gamma$ for every $\lambda \in[h(\alpha),+\infty)$. Then,

$s_{1}(\lambda)=\frac{\frac{s_{1}(\lambda) s_{2}(\lambda)\left|r_{\alpha}(\lambda)\right|}{\rho(\alpha)}}{\frac{s_{2}(\lambda)\left|r_{\alpha}(\lambda)\right|}{\rho(\alpha)}} \leq \frac{s_{2}(\lambda)}{\gamma} \frac{s_{1}(\lambda)\left|r_{\alpha}(\lambda)\right|}{\rho(\alpha)} \quad \forall \lambda \in[h(\alpha),+\infty), \forall \alpha \in\left(0, \alpha_{0}\right)$. 
On the other hand, since $\left(s_{1}, \rho\right)$ is a strong source-order pair, there exist positive constants $k$ and $\varepsilon$ such that

$$
\frac{s_{1}(\lambda)\left|r_{\alpha}(\lambda)\right|}{\rho(\alpha)} \leq k \quad \forall \lambda>0, \forall \alpha \in(0, \varepsilon) .
$$

From (15) and (16) it follows that

$$
s_{1}(\lambda) \leq \frac{k}{\gamma} s_{2}(\lambda) \quad \forall \lambda \in[h(\alpha),+\infty), \forall \alpha \in(0, \varepsilon) .
$$

Since $\lim _{\alpha \rightarrow 0^{+}} h(\alpha)=0$ we have that $s_{1}(\lambda) \leq \frac{k}{\gamma} s_{2}(\lambda)$ for every $\lambda>0$. Analogously, by interchanging $s_{1}$ and $s_{2}$ it follows that there exists $\tilde{k}>0$ such that $s_{2}(\lambda) \leq \tilde{k} s_{1}(\lambda)$ for every $\lambda>0$ and therefore, $s_{1} \approx s_{2}$.

Suppose now that $s_{\rho} \in \mathcal{S}$. Since $\rho$ is optimal qualification of $\left\{g_{\alpha}\right\}$ it follows from Theorem 2.2 that $s_{\rho}$ verifies (6) and (12). Then, $s_{\rho}$ is the unique function such that $\left(s_{\rho}, \rho\right)$ is a strong source-order pair and $\left(\rho, s_{\rho}\right)$ is an order-source pair for $\left\{g_{\alpha}\right\}$.

The following is a result about the uniqueness of the order.

Theorem 2.4. If $\left(s, \rho_{1}\right)$ and $\left(s, \rho_{2}\right)$ are strong source-order pairs for $\left\{g_{\alpha}\right\}$ and there exists $\lim _{\alpha \rightarrow 0^{+}} \frac{\rho_{1}(\alpha)}{\rho_{2}(\alpha)}$, then $\rho_{1} \approx \rho_{2}$.

Proof. Suppose that $\left(s, \rho_{1}\right)$ and $\left(s, \rho_{2}\right)$ are strong source-order pairs for $\left\{g_{\alpha}\right\}$. We will first show that $\limsup _{\alpha \rightarrow 0^{+}} \frac{\rho_{1}(\alpha)}{\rho_{2}(\alpha)}>0$. Suppose that

$$
\limsup _{\alpha \rightarrow 0^{+}} \frac{\rho_{1}(\alpha)}{\rho_{2}(\alpha)}=0
$$

Since $\left(s, \rho_{1}\right)$ is a strong source-order pair we have that

$$
\frac{s(\lambda)\left|r_{\alpha}(\lambda)\right|}{\rho_{1}(\alpha)}=O(1) \text { for } \alpha \rightarrow 0^{+}, \forall \lambda>0
$$

and

$$
0<\limsup _{\alpha \rightarrow 0^{+}} \frac{s(\lambda)\left|r_{\alpha}(\lambda)\right|}{\rho_{2}(\alpha)}=\limsup _{\alpha \rightarrow 0^{+}} \frac{s(\lambda)\left|r_{\alpha}(\lambda)\right|}{\rho_{1}(\alpha)} \frac{\rho_{1}(\alpha)}{\rho_{2}(\alpha)} .
$$

It follows from (17) and (18) that the limsup on the right-hand side of the previous expression must be equal to zero, which is a contradiction. Then, $\underset{\alpha \rightarrow 0^{+}}{\lim \sup } \frac{\rho_{1}(\alpha)}{\rho_{2}(\alpha)}>0$. Similarly, it is shown that $\limsup _{\alpha \rightarrow 0^{+}} \frac{\rho_{2}(\alpha)}{\rho_{1}(\alpha)}>0$. Since there exists $\lim _{\alpha \rightarrow 0^{+}} \frac{\rho_{1}(\alpha)}{\rho_{2}(\alpha)}$, we then have that $0<\lim _{\alpha \rightarrow 0^{+}} \frac{\rho_{1}(\alpha)}{\rho_{2}(\alpha)}<+\infty$ and $0<\lim _{\alpha \rightarrow 0^{+}} \frac{\rho_{2}(\alpha)}{\rho_{1}(\alpha)}<+\infty$. Then, $\rho_{1} \preceq \rho_{2}$ and $\rho_{2} \preceq \rho_{1}$, that is, $\rho_{1} \approx \rho_{2}$, as we wanted to show. 


\section{Examples}

In this section we present several examples which illustrate the different qualification levels previously introduced as well as the relationships between them and with the concept of classical qualification and the qualification introduced in [6]. Although some of these examples are only of academic interest and nature, they do serve to show the existence of regularization methods possessing qualification in each one of the levels introduced in this article.

Example 1. Tikhonov-Phillips regularization method $\left\{g_{\alpha}\right\}$, where $g_{\alpha}(\lambda) \doteq \frac{1}{\lambda+\alpha}$ has classical qualification of order $\mu_{0}=1([2])$. We will see that $\rho(\alpha)=\alpha$ is optimal qualification in the sense of Definition $2.4 \mathrm{iii})$. In fact, for $\lambda>0, r_{\alpha}(\lambda)=\frac{\alpha}{\alpha+\lambda}$ and if $\rho(\alpha)=\alpha$ then $s_{\rho}(\lambda)=\liminf _{\alpha \rightarrow 0^{+}} \frac{\rho(\alpha)}{\left|r_{\alpha}(\lambda)\right|}=\lim _{\alpha \rightarrow 0}(\lambda+\alpha)=\lambda>0$, that is, $s_{\rho}$ verifies (12). Also since

$$
\frac{s_{\rho}(\lambda)\left|r_{\alpha}(\lambda)\right|}{\rho(\alpha)}=\frac{\lambda}{\lambda+\alpha} \geq \frac{1}{2} \quad \forall \lambda \in[\alpha,+\infty),
$$

we have that $s_{\rho}$ verifies (6). From Theorem 2.2 it then follows that $\rho(\alpha)=\alpha$ is optimal qualification of $\left\{g_{\alpha}\right\}$.

Example 2. Let $\left\{g_{\alpha}\right\}$ be the family of functions associated to the truncated singular value decomposition (TSVD),

$$
g_{\alpha}(\lambda) \doteq \begin{cases}\frac{1}{\lambda}, & \text { if } \lambda \in[\alpha,+\infty) \\ 0, & \text { if } \lambda \in[0, \alpha) .\end{cases}
$$

It follows that $\mu_{0}=+\infty$, where $\mu_{0}$ is as in Definition 1.1. Therefore, TSVD does not have classical qualification. In this case we have that

$$
r_{\alpha}(\lambda)= \begin{cases}0, & \text { if } \lambda \in[\alpha,+\infty) \\ 1, & \text { if } \lambda \in[0, \alpha) .\end{cases}
$$

Let $h(\alpha)=\alpha$ and $\rho \in \mathcal{O}$. Then

$$
\sup _{\lambda \in[h(\alpha),+\infty)}\left|r_{\alpha}(\lambda)\right|=\sup _{\lambda \geq \alpha}\left|r_{\alpha}(\lambda)\right|=0 \leq \rho(\alpha) \quad \text { for every } \alpha \in\left(0, \alpha_{0}\right) .
$$

Then, it follows from Theorem 2.1.a) that any function $\rho \in \mathcal{O}$ is weak qualification of the method. However, TSVD does not have strong qualification. In fact, for any function $\rho \in \mathcal{O}$ we have that $s_{\rho}(\lambda)=\liminf _{\alpha \rightarrow 0^{+}} \frac{\rho(\alpha)}{\left|r_{\alpha}(\lambda)\right|}=+\infty$ for every $\lambda>0$. Proposition 2.1 implies then that $\rho$ is not strong qualification of the method. In [6] it was observed that TSVD has arbitrary qualification in the sense of Definition 1.2.

Example 3. For $\alpha \in\left(0, \alpha_{0}\right)$ we define

$$
g_{\alpha}(\lambda) \doteq \frac{1-e^{-\frac{1}{\alpha}}}{\lambda+e^{-\frac{1}{\alpha}}}, \quad \text { for every } \lambda \in[0,+\infty) .
$$


It can be immediately verified that $\left\{g_{\alpha}\right\}$ satisfies the hypotheses $H 1-H 3$ and therefore is a SRM. Since $r_{\alpha}(\lambda)=\frac{1+\lambda}{1+\lambda e^{\frac{1}{\alpha}}}$ for all $\lambda \in[0,+\infty)$, it follows that for every $\mu>0$,

$$
\frac{\left|r_{\alpha}(\lambda)\right| \lambda^{\mu}}{\alpha^{\mu}}=\frac{(1+\lambda) \lambda^{\mu}}{\lambda e^{\frac{1}{\alpha}} \alpha^{\mu}+\alpha^{\mu}}=o(1) \text { for } \alpha \rightarrow 0^{+} \text {for every } \lambda \in[0,+\infty) .
$$

Then, $\left\{g_{\alpha}\right\}$ does not have classical qualification (more precisely $\mu_{0}=+\infty$, where $\mu_{0}$ is as in Definition 1.1).

We will now show that $\rho(\alpha)=e^{-\frac{1}{\alpha}}$ is optimal qualification of $\left\{g_{\alpha}\right\}$. Since $s_{\rho}(\lambda)=\liminf _{\alpha \rightarrow 0^{+}} \frac{\rho(\alpha)}{\left|r_{\alpha}(\lambda)\right|}=\frac{\lambda}{1+\lambda} \in(0,+\infty)$ for every $\lambda>0$, it follows from Proposition 2.1 that $\rho$ is strong qualification of $\left\{g_{\alpha}\right\}$. Moreover since

$$
\frac{s_{\rho}(\lambda)\left|r_{\alpha}(\lambda)\right|}{\rho(\alpha)}=\frac{\lambda}{\lambda+e^{-\frac{1}{\alpha}}} \geq \frac{1}{2} \quad \forall \lambda \in\left[e^{-\frac{1}{\alpha}},+\infty\right)
$$

it follows that $s_{\rho}$ verifies (6). Theorem 2.2 then implies that $\rho(\alpha)=e^{-\frac{1}{\alpha}}$ is optimal qualification of $\left\{g_{\alpha}\right\}$.

Example 4. For $\alpha \in\left(0, \alpha_{0}\right)$ with $\alpha_{0}<e^{-1}$, define

$$
g_{\alpha}(\lambda) \doteq \frac{1+(\ln \alpha)^{-1}}{\lambda-(\ln \alpha)^{-1}}, \quad \text { for every } \lambda \in[0,+\infty) .
$$

Clearly, $\left\{g_{\alpha}\right\}$ satisfies hypotheses $H 1-H 3$ and therefore is a SRM. Since $r_{\alpha}(\lambda)=\frac{1+\lambda}{1-\lambda \ln \alpha}$ for all $\lambda \in[0,+\infty)$, it follows that for every $\mu>0$,

$$
\frac{\left|r_{\alpha}(\lambda)\right| \lambda^{\mu}}{\alpha^{\mu}}=\frac{(1+\lambda) \lambda^{\mu}}{\alpha^{\mu}-\lambda \alpha^{\mu} \ln \alpha} \rightarrow+\infty \text { for } \alpha \rightarrow 0^{+} \text {for every } \lambda \in[0,+\infty) .
$$

Then, $\mu_{0}=0$ and therefore $\left\{g_{\alpha}\right\}$ does not have classical qualification.

However, we will show that $\rho(\alpha)=-(\ln \alpha)^{-1}$ is optimal qualification of $\left\{g_{\alpha}\right\}$. In fact, since $s_{\rho}(\lambda)=\liminf _{\alpha \rightarrow 0^{+}} \frac{\rho(\alpha)}{\left|r_{\alpha}(\lambda)\right|}=\lim _{\alpha \rightarrow 0} \frac{\lambda-(\ln \alpha)^{-1}}{1+\lambda}=\frac{\lambda}{1+\lambda} \in(0,+\infty)$ for every $\lambda>0$ and

$$
\frac{s_{\rho}(\lambda)\left|r_{\alpha}(\lambda)\right|}{\rho(\alpha)}=\frac{\lambda}{\lambda-(\ln \alpha)^{-1}} \geq \frac{1}{2} \quad \forall \lambda \in\left[-(\ln \alpha)^{-1},+\infty\right),
$$

it follows from Theorem 2.2 that $\rho(\alpha)=-(\ln \alpha)^{-1}$ is optimal qualification of $\left\{g_{\alpha}\right\}$.

Example 5. Let $\left\{g_{\alpha}\right\}$ be the Tikhonov-Phillips regularization method, which, as previously mentioned, it has classical qualification of order $\mu_{0}=1$. In Example 1 we saw that $\rho(\alpha)=\alpha$ is optimal qualification of this method and therefore it is also weak qualification of it. Since $\alpha \preceq \alpha^{\frac{1}{2}}$ it follows from Definition 2.4.i) and Observation 2.a) that $\rho^{*}(\alpha)=\alpha^{\frac{1}{2}}$ is also weak qualification. However, $\rho^{*}$ is not strong qualification of the method. In fact, for any $s \in \mathcal{S}$, we have that

$$
\limsup _{\alpha \rightarrow 0^{+}} \frac{s(\lambda)\left|r_{\alpha}(\lambda)\right|}{\rho^{*}(\alpha)}=\limsup _{\alpha \rightarrow 0^{+}} \frac{s(\lambda) \alpha^{\frac{1}{2}}}{\alpha+\lambda}=0 \quad \forall \lambda>0 .
$$

Example 6. Let $\left\{g_{\alpha}\right\}$ be the SRM defined in Example 4. This method does not have classical qualification since $\mu_{0}=0$. We proved that $-(\ln \alpha)^{-1}$ is optimal qualification and therefore, it is also weak qualification. Since $-(\ln \alpha)^{-1} \preceq(-\ln \alpha)^{-\frac{1}{2}}$, 
just like in the previous example, it follows immediately that $\rho(\alpha)=(-\ln \alpha)^{-\frac{1}{2}}$ is weak qualification. Let us show now that $\rho$ is not strong qualification of the method. For any $s \in \mathcal{S}$, we have that

$$
\limsup _{\alpha \rightarrow 0^{+}} \frac{s(\lambda)\left|r_{\alpha}(\lambda)\right|}{\rho(\alpha)}=\limsup _{\alpha \rightarrow 0^{+}} \frac{s(\lambda)(1+\lambda)}{(1-\lambda \ln \alpha)(-\ln \alpha)^{-\frac{1}{2}}}=0 \quad \forall \lambda>0 .
$$

It is important to observe that if $\rho(\alpha)=\alpha^{\mu}$ is strong qualification of a SRM then it follows immediately from the definition of strong source-order pair that the method has classical qualification of order $\mu$. The converse, however, is not true as the next example shows. Hence it is the weak and not the strong qualification what generalizes the classical notion of this concept.

Example 7. For $\alpha \in\left(0, \alpha_{0}\right)$ with $\alpha_{0}<1 / 2$ define

$$
h_{\alpha}(\lambda) \doteq \frac{\alpha}{\alpha+\ln \left(\frac{\alpha}{\alpha+\lambda}\right)}
$$

and

$$
g_{\alpha}(\lambda) \doteq \begin{cases}\frac{1-h_{\alpha}(\lambda)}{\lambda+h_{\alpha}(\lambda)}, & \text { if } \lambda \in[2 \alpha,+\infty) \\ \frac{1-h_{\alpha}(2 \alpha)}{2 \alpha+h_{\alpha}(2 \alpha)}=\left(2 \alpha-\frac{\alpha+2 \alpha^{2}}{\ln 3}\right)^{-1}, & \text { if } \lambda \in[0,2 \alpha) .\end{cases}
$$

In this case,

$$
r_{\alpha}(\lambda) \doteq \begin{cases}\frac{\alpha(1+\lambda)}{\lambda \ln \left(\frac{\alpha}{\alpha+\lambda}\right)+\alpha(1+\lambda)}, & \text { if } \lambda \in[2 \alpha,+\infty) \\ 1-\lambda\left(2 \alpha-\frac{\alpha+2 \alpha^{2}}{\ln 3}\right)^{-1}, & \text { if } \lambda \in[0,2 \alpha)\end{cases}
$$

One can immediately show that $\left\{g_{\alpha}\right\}$ is a SRM with classical qualification of order $\mu_{0}=1$. However, $\rho(\alpha)=\alpha$ is not strong qualification of the method. In fact, for any $s \in \mathcal{S}$, we can see that

$$
\frac{s(\lambda)\left|r_{\alpha}(\lambda)\right|}{\alpha}=o(1) \text { for } \alpha \rightarrow 0^{+}, \forall \lambda \geq 0
$$

and therefore condition (5) is not satisfied.

SRMs possessing strong but not optimal qualification, have very peculiar properties. Thus for instance, it is possible to show that if $\rho$ is strong qualification which is not optimal, then $\forall \lambda>0$, the function $\frac{s_{\rho}(\lambda)\left|r_{\alpha}(\lambda)\right|}{\rho(\alpha)}$ it is not of bounded variation as a function of $\alpha$ in any neighborhood of $\alpha=0$. Even so, the following three examples show the existence of SRM having strong but not optimal qualification and they show that strong qualification in no case implies optimal qualification.

Example 8. Given $k \in \mathbb{R}^{+}$, for $\alpha, \lambda>0$ define

$$
g_{\alpha}^{k}(\lambda) \doteq \lambda^{-1}\left(1-e^{-\frac{\lambda}{\alpha}}\right)-\alpha^{k} \lambda^{-3 / 2}\left|\sin \left(\lambda^{\frac{3}{2}} / \alpha\right)\right|
$$


so that $r_{\alpha}^{k}(\lambda)=e^{-\frac{\lambda}{\alpha}}+\alpha^{k} \lambda^{-1 / 2}\left|\sin \left(\lambda^{\frac{3}{2}} / \alpha\right)\right|$. It can be immediately checked that $\left\{g_{\alpha}^{k}\right\}$ is a SRM with classical qualification of order $k$. With $\rho(\alpha)=\alpha^{k}$ we have that $\forall \lambda>0$,

$$
\begin{aligned}
& s_{\rho}(\lambda)=\liminf _{\alpha \rightarrow 0^{+}} \frac{\alpha^{k}}{e^{-\frac{\lambda}{\alpha}}+\alpha^{k} \lambda^{-1 / 2}\left|\sin \left(\lambda^{\frac{3}{2}} / \alpha\right)\right|} \\
& =\frac{1}{\limsup _{\alpha \rightarrow 0^{+}}\left(\alpha^{-k} e^{-\frac{\lambda}{\alpha}}+\lambda^{-1 / 2}\left|\sin \left(\lambda^{\frac{3}{2}} / \alpha\right)\right|\right)} \\
& =\lambda^{1 / 2} \text {. }
\end{aligned}
$$

Since $s_{\rho}(\lambda)=\lambda^{1 / 2} \in \mathcal{S}$, from Proposition 2.1 it follows that $\left(s_{\rho}, \rho\right)$ is a strong sourceorder pair and $\rho(\alpha)=\alpha^{k}$ is strong qualification of the method. However, for every $\lambda>0$,

$$
\liminf _{\alpha \rightarrow 0^{+}} \frac{s_{\rho}(\lambda)\left|r_{\alpha}(\lambda)\right|}{\rho(\alpha)}=\liminf _{\alpha \rightarrow 0^{+}}\left[\lambda^{1 / 2} \alpha^{-k} e^{-\frac{\lambda}{\alpha}}+\left|\sin \left(\frac{\lambda^{\frac{3}{2}}}{\alpha}\right)\right|\right]=0 .
$$

Therefore equation (6) does not hold and $\rho(\alpha)=\alpha^{k}$ is not optimal qualification of the method.

Example 9. For $\alpha, \lambda>0$ define $g_{\alpha}(\lambda)$ as follows:

$$
g_{\alpha}(\lambda) \doteq \lambda^{-1}\left(1-e^{-\frac{\lambda}{\alpha}}\right)-e^{-\frac{1}{\sqrt{\alpha}}} \lambda^{-3 / 2}\left|\sin \left(\lambda^{\frac{3}{2}} / \alpha\right)\right|,
$$

so that

$$
r_{\alpha}(\lambda)=e^{-\frac{\lambda}{\alpha}}+e^{-\frac{1}{\sqrt{\alpha}}} \lambda^{-1 / 2}\left|\sin \left(\lambda^{\frac{3}{2}} / \alpha\right)\right|
$$

It can be immediately verified that $\left\{g_{\alpha}\right\}$ is a SRM which does not have classical qualification $\left(\mu_{0}=\infty\right)$. However, with $\rho(\alpha) \doteq e^{-\frac{1}{\sqrt{\alpha}}}$ we have that

$$
\begin{aligned}
s_{\rho}(\lambda) & =\liminf _{\alpha \rightarrow 0^{+}} \frac{\rho(\alpha)}{r_{\alpha}(\lambda)} \\
& =\frac{1}{\limsup _{\alpha \rightarrow 0^{+}}\left[e^{-\frac{\lambda}{\alpha}+\frac{1}{\sqrt{\alpha}}}+\lambda^{-1 / 2}\left|\sin \left(\lambda^{\frac{3}{2}} / \alpha\right)\right|\right]} \\
& =\lambda^{\frac{1}{2}} .
\end{aligned}
$$

Since $s_{\rho}(\lambda)=\lambda^{1 / 2} \in \mathcal{S}$, by Proposition $2.1\left(s_{\rho}, \rho\right)$ is a strong source-order pair and $\rho(\alpha)=e^{-1 / \sqrt{\alpha}}$ is strong qualification of the method. However, $\forall \lambda>0$ we have that

$$
\liminf _{\alpha \rightarrow 0^{+}} \frac{s_{\rho}(\lambda)\left|r_{\alpha}(\lambda)\right|}{\rho(\alpha)}=\liminf _{\alpha \rightarrow 0^{+}}\left(\lambda^{1 / 2} e^{\frac{1}{\sqrt{\alpha}}-\frac{\lambda}{\alpha}}+\left|\sin \left(\lambda^{\frac{3}{2}} / \alpha\right)\right|\right)=0,
$$

and therefore (6) does not hold and $\rho(\alpha)=e^{-\frac{1}{\sqrt{\alpha}}}$ is not optimal qualification of the method.

Example 10. For $0<\alpha<1$ and $\lambda>0$ define

$$
g_{\alpha}(\lambda) \doteq \lambda^{-1}\left(1-e^{-\frac{\lambda}{\alpha}}\right)+(\ln \alpha)^{-1} \lambda^{-3 / 2}\left|\sin \left(\lambda^{\frac{3}{2}} / \alpha\right)\right|,
$$


so that

$$
r_{\alpha}(\lambda)=e^{-\frac{\lambda}{\alpha}}-(\ln \alpha)^{-1} \lambda^{-1 / 2}\left|\sin \left(\lambda^{\frac{3}{2}} / \alpha\right)\right| .
$$

Just like in Examples 8 and 9 it can be easily checked that $\left\{g_{\alpha}\right\}$ is a SRM which does not have classical qualification $\left(\mu_{0}=0\right)$, that $\rho(\alpha)=\frac{-1}{\ln \alpha}$ is strong but not optimal qualification of the method and that $\left(s_{\rho}, \rho\right)$ is a strong source-order pair with $s_{\rho}(\lambda)=\lambda^{\frac{1}{2}}$.

Note that examples 2, 3, 4, 6, 9 and 10 correspond to SRMs which do not have classical qualification but, however, they do have generalized qualification, falling in some of its three different levels. Also Landweber's method and Showalter's method, which as previously pointed out do not have classical qualification (in both cases $\left.\mu_{0}=\infty\right)$, are SRMs defined by $g_{\alpha}(\lambda) \doteq \frac{1}{\lambda}\left(1-(1-\mu \lambda)^{\frac{1}{\alpha}}\right)$ (where $\left.\alpha \leq 1, \mu<\frac{1}{\|T\|^{2}}\right)$ and $g_{\alpha}(\lambda) \doteq \frac{1}{\lambda}\left(1-e^{-\frac{\lambda}{\alpha}}\right)$, respectively. It can be easily proved, by using Theorem 2.1 , that $\rho(\alpha)=\left(1-\mu \alpha^{\frac{1}{2}}\right)^{\frac{1}{\alpha}}$ is weak qualification of Landweber's method and $\rho(\alpha)=e^{-\frac{1}{\sqrt{\alpha}}}$ is weak qualification of Showalter's method. However, in this last case it can be easily shown that $\rho(\alpha)=e^{-\frac{1}{\sqrt{\alpha}}}$ does not satisfy condition (3) and therefore $\rho(\alpha)$ is not qualification in the sense of Definition 1.2.

The different qualification levels introduced in this article and the relationships between them are visualized in Figure 1.

\section{Orders of convergence, converse results and maximal source sets}

The generalization of the concept of qualification of a SRM introduced in the previous sections is strongly related with and it has a broad spectrum of applications in the context of orders of convergence, converse results and maximal source sets for inverse ill-posed problems. We present next some results in this direction. However, we point out that this is not the main objective of the present article. For that reason, some of this results will be stated without proof. More detailed results in this regard will appear in a forthcoming article.

Let $X, Y$ be infinite dimensional Hilbert spaces and $T: X \rightarrow Y$ a bounded, linear invertible operator such that $\mathcal{R}(T)$ is not closed. For $s \in \mathcal{S}$, the set $\mathcal{R}\left(s\left(T^{*} T\right)\right.$ ), will be referred to as the "source set associated to the function $s$ and the operator $T$ ". In all that follows, the hypothesis $s \in \mathcal{S}$ can be replaced by $s$ continuous on $\sigma\left(T^{*} T\right)$ and $s \in \mathcal{M}_{0}$, where $\mathcal{M}_{0}$ is the set of all functions $f: \mathbb{R} \rightarrow \mathbb{R}_{0}^{+}$which are measurable with respect to the measures $d\left\|E_{\lambda} x\right\|^{2}$ for every $x \in X$.

The following direct result, whose proof follows immediately from the concept of weak source-order pair, states that if the exact solution $x^{\dagger}$ of the problem $T x=y$ belongs to the source set $\mathcal{R}\left(s\left(T^{*} T\right)\right)$ and $(s, \rho)$ is a weak source-order pair for $\left\{g_{\alpha}\right\}$, then the regularization error $\left\|R_{\alpha} y-x^{\dagger}\right\|$ has order of convergence $\rho(\alpha)$. For brevity reasons we do not give the proof here. 

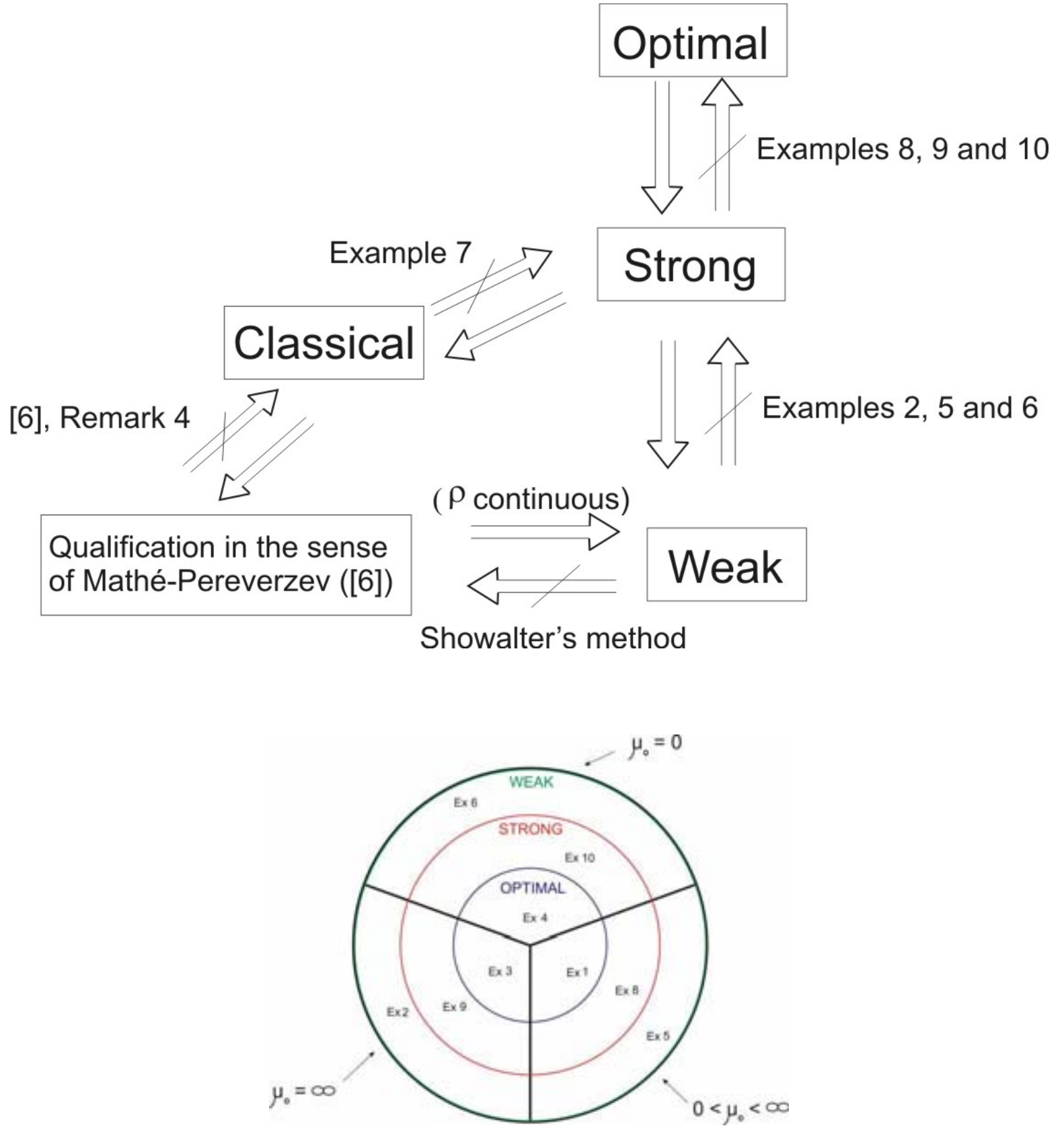

Figure 1. Relationships between the different qualification levels, the classical qualification and the qualification defined in [6].

Theorem 4.1. Let $\rho \in \mathcal{O}$ be weak qualification of $\left\{g_{\alpha}\right\}$ and $s \in \mathcal{S}$ such that $(s, \rho)$ is a weak source-order pair for $\left\{g_{\alpha}\right\}$. If $x^{\dagger} \doteq T^{\dagger} y \in \mathcal{R}\left(s\left(T^{*} T\right)\right)$ then $\left\|\left(R_{\alpha}-T^{\dagger}\right) y\right\|=$ $O(\rho(\alpha))$ for $\alpha \rightarrow 0^{+}$.

It is important to note here that the previous result can be viewed as a generalization of Theorem 4.3 in [2], to the case of SRM with weak qualification and general source sets. In fact, that result corresponds to the particular case in which $\left\{g_{\alpha}\right\}$ has classical qualification of order $\mu$. 
The following converse result states that if the regularization error has order of convergence $\rho(\alpha)$ and $(\rho, s)$ is an order-source pair, then the exact solution belongs to the source set given by the range of the operator $s\left(T^{*} T\right)$.

Theorem 4.2. If $(\rho, s)$ is an order-source pair for $\left\{g_{\alpha}\right\}$ and $\left\|\left(R_{\alpha}-T^{\dagger}\right) y\right\|=O(\rho(\alpha))$ for $\alpha \rightarrow 0^{+}$, then $x^{\dagger} \in \mathcal{R}\left(s\left(T^{*} T\right)\right)$.

Proof. The proof follows immediately from the definition of order-source pair for the $\operatorname{SRM}\left\{g_{\alpha}\right\}$.

It is interesting to note that Theorem 4.2 can also be viewed as a generalization of Theorem 4.11 in [2]. In fact, this corresponds to the particular case in which $s(\lambda) \doteq \lambda^{\mu}$ y $\rho(\alpha) \doteq \alpha^{\mu}$. If moreover $\rho$ is optimal qualification then the reciprocal of Theorem 4.2 also holds. This is proved in the following theorem.

Theorem 4.3. If $\rho$ is optimal qualification of $\left\{g_{\alpha}\right\}$ and $s_{\rho} \in \mathcal{S}$, then $\left\|\left(R_{\alpha}-T^{\dagger}\right) y\right\|=$ $O(\rho(\alpha))$ for $\alpha \rightarrow 0^{+}$if and only if $x^{\dagger} \in \mathcal{R}\left(s_{\rho}\left(T^{*} T\right)\right)$.

Proof. Let $\rho$ be optimal qualification of $\left\{g_{\alpha}\right\}$ and $s_{\rho} \in \mathcal{S}$. Then by Theorem 2.3, $\left(\rho, s_{\rho}\right)$ is an order-source pair for $\left\{g_{\alpha}\right\}$ and since $\left\|\left(R_{\alpha}-T^{\dagger}\right) y\right\|=O(\rho(\alpha))$ for $\alpha \rightarrow 0^{+}$, it follows from Theorem 4.2 that $x^{\dagger} \in \mathcal{R}\left(s_{\rho}\left(T^{*} T\right)\right)$.

Conversely, if $x^{\dagger} \in \mathcal{R}\left(s_{\rho}\left(T^{*} T\right)\right)$, since by virtue of Theorem $2.3\left(s_{\rho}, \rho\right)$ is a strong source-order pair, Theorem 4.1 implies that $\left\|\left(R_{\alpha}-T^{\dagger}\right) y\right\|=O(\rho(\alpha))$ for $\alpha \rightarrow 0^{+}$.

An important result regarding existence and maximality of source sets is the following: if $\rho$ is strong qualification of a SRM and $s_{\rho} \in \mathcal{S}$ it follows from Proposition 2.2 that $\mathcal{R}\left(s_{\rho}\left(T^{*} T\right)\right)$ is a maximal source set where $\rho$ is order of convergence of the regularization error. More precisely we have the following result.

Theorem 4.4. Let $\rho \in \mathcal{O}$ be strong qualification of $\left\{g_{\alpha}\right\}$ such that $s_{\rho} \in \mathcal{S}$ and $s \in \mathcal{S}$. If $(s, \rho)$ is a strong source-order pair for $\left\{g_{\alpha}\right\}$ and $\mathcal{R}\left(s\left(T^{*} T\right)\right) \supset \mathcal{R}\left(s_{\rho}\left(T^{*} T\right)\right)$ then $\mathcal{R}\left(s\left(T^{*} T\right)\right)=\mathcal{R}\left(s_{\rho}\left(T^{*} T\right)\right)$.

Proof. Under the hypotheses of the Proposition 2.2, there exists $k>0$ such that $s(\lambda) \leq k s_{\rho}(\lambda)$ for every $\lambda>0$, which implies that $\mathcal{R}\left(s\left(T^{*} T\right)\right) \subset \mathcal{R}\left(s_{\rho}\left(T^{*} T\right)\right)$.

If moreover $\rho$ is optimal qualification the following stronger result is obtained.

Theorem 4.5. If $\rho \in \mathcal{O}$ is optimal qualification of $\left\{g_{\alpha}\right\}$ and $s_{\rho} \in \mathcal{S}$, then $\mathcal{R}\left(s_{\rho}\left(T^{*} T\right)\right)$ is the only source set where $\rho$ is order of convergence of the regularization error of $\left\{g_{\alpha}\right\}$.

Proof. This result follows immediately from Theorem 2.3.

\section{Examples:}

1. For the Tikhonov-Phillips regularization method the only source set where $\rho(\alpha)=\alpha$ is optimal qualification is $\mathcal{R}\left(s_{\rho}\left(T^{*} T\right)\right)=\mathcal{R}\left(T^{*} T\right)$, since in this case $s_{\rho}(\lambda)=\lambda$. 
2. In Example 3 of Section 3 we saw that $\rho(\alpha)=e^{-\frac{1}{\alpha}}$ is optimal qualification of $\left\{g_{\alpha}\right\}$ and $s_{\rho}(\lambda)=\frac{\lambda}{1+\lambda}$. Since $\frac{\lambda}{1+\lambda} \approx \lambda$ it follows that $\mathcal{R}\left(s_{\rho}\left(T^{*} T\right)\right)=\mathcal{R}\left(T^{*} T\right)$ is the only source set where $\rho$ is order of convergence of the regularization error.

3. In Example 8 of the previous section, for $\rho(\alpha)=\alpha^{k}$ we have that $s_{\rho}(\lambda)=\lambda^{1 / 2}$. Since $\rho$ is strong qualification of this SRM, it follows that $\mathcal{R}\left(s_{\rho}\left(T^{*} T\right)\right)=\mathcal{R}\left(T^{*} T\right)^{1 / 2}$ is a maximal source set where $\rho(\alpha)$ is order of convergence of the regularization error.

4. As pointed out at the end of Section $3, \rho(\alpha)=e^{-\frac{1}{\sqrt{\alpha}}}$ is weak qualification of Showalter's method. It can be easily shown that for every $s \in \mathcal{S},(s, \rho)$ is a weak sourceorder pair for the method. Therefore, it follows from Theorem 4.1 that the regularization error $\left\|R_{\alpha} y-x^{\dagger}\right\|$ has order of convergence $\rho(\alpha)=e^{-\frac{1}{\sqrt{\alpha}}}$ whenever $x^{\dagger} \in \bigcup_{s \in \mathcal{S}} \mathcal{R}\left(s\left(T^{*} T\right)\right)$.

5. Same as 4. happens with Landweber's method and $\rho(\alpha)=(1-\mu \sqrt{\alpha})^{\frac{1}{\alpha}}$.

\section{Conclusions}

In this article we have extended the definition of qualification for spectral regularization methods introduced by Mathé and Pereverzev in [6]. This extension was constructed bearing in mind the concept of qualification as the optimal order of convergence of the regularization error that a method can achieve ([2], [5], [6], [11]). Three different levels of generalized qualification were introduced: weak, strong and optimal. In particular, the first of these levels extends the definition introduced in [6] and a SRM having weak qualification which is not qualification in the sense of Definition 1.2 was shown. Sufficient conditions for a SRM to have weak qualification were provided, as well as necessary and sufficient conditions for a given order of convergence to be strong or optimal qualification. Examples of all three qualification levels were provided and the relationships between them as well as with the classical concept of qualification and the qualification introduced in [6] were shown. Several SRMs having generalized qualification in each one of the three levels and not having classical qualification were presented. In particular, it was shown that the well known TSVD, Showalter's and Landweber's methods do have weak qualification. Finally several implications of this theory in the context of orders of convergence, converse results and maximal source sets for inverse ill-posed problems, were briefly shown. More detailed results on these implications will appear in a forthcoming article.

\section{References}

[1] DAUTRAY R., and LIONS J.-L.: Spectral Theory and Applications, volume 3 of Mathematical Analysis and Numerical Methods for Science and Technology. Springer (1990).

[2] ENGL, H. W., HANKE, M., and NEUBAUER A.: Regularization of inverse problems, volume 375 of Mathematics and its Applications. Kluwer Academic Publishers Group, Dordrecht (1996).

[3] ENGL, H. W., KUNISCH, K., and NEUBAUER, A.: Convergence rates for Tikhonov regularization of nonlinear ill-posed problems. Inverse Problems, 5(4), 523-540, (1989).

[4] LUECKE, G. R., and HICKEY, K. R.: Convergence of approximate solutions of an operator equation. Houston Journal of Mathematics, 11(3), 345-354 (1985). 
[5] MATHÉ, P.: Saturation of regularization methods for linear ill-posed problems in Hilbert spaces. SIAM J. Numer. Anal., 42(3), 968-973 (electronic) (2004).

[6] MATHÉ, P., and PEREVERZEV, S. V.: Geometry of linear ill-posed problems in variable Hilbert scales. Inverse Problems, 19(3), 789-803 (2003).

[7] NEUBAUER, A.: On converse and saturation results for regularization methods. In Beiträge zur angewandten Analysis und Informatik, pages 262-270. Shaker, Aachen (1994).

[8] NEUBAUER, A.: On converse and saturation results for Tikhonov regularization of linear illposed problems. SIAM J. Numer. Anal., 34(2), 517-527 (1997).

[9] SEIDMAN, T. I.: Nonconvergence results for the application of least-squares estimation to illposed problems. J. Optim. Theory Appl., 30(4), 535-547 (1980).

[10] SPIES, R. D., and TEMPERINI, K. G.: Arbitrary divergence speed of the least-squares method in infinite-dimensional inverse ill-posed problems. Inverse Problems, 22(2), 611-626 (2006).

[11] VAINIKKO, G. M.: The discrepancy principle for a class of regularization methods. U.S.S.R. Comput. Maths. Math. Phys., 22(3), 1-19 (1982).

[12] VAINIKKO, G. M., and HÄMARIK, U.: Projection Methods and self-regularization in ill-posed problems. Soviet Mathematics, 29(10), 1-20 (1985). 